\title{
The systematic analysis of the transformation between the German geodetic reference system (DHDN, DHHN) and the ETRF system (DREF91)
}

\author{
Jianqing Cai* \\ Geodetic Institute, RWTH Aachen, Templergraben 55, 52062 Aachen, Germany
}

(Received January 11, 2000; Revised August 9, 2000; Accepted August 25, 2000)

\begin{abstract}
The 69 colocated sites in the area of the West Germany are used to estimate the transformation parameters with the seven parameter similarity transformation model. According to the solutions of transformation parameters and corresponding residuals between DHDN and ETRF, detail analyses for global and regional transformation are performed, which show that the global accuracy of the transformation between DHDN and ETRF is $\pm 5 \mathrm{~m}$ with WRMS (weighted root mean square) of $\pm 0.64 \mathrm{~m}$ and regional transformations have much better fitting. Through the comparisons of estimated parameters of each state, the systematic differences in definition and observation among regional systems of DHDN have been confirmed. Practical usage of 7 parameter similarity transformation and some suggestions are pointed out.
\end{abstract}

\section{The Introduction of German National Coordi- nate System (DHDN, DHHN)}

The Primary Triangulation Net of the West Germany (DHDN) was emerged between 1870 and 1950 from the combination of several individual networks. The Bessel ellipsoid (1841) served as a reference surface. The origin point is in Rauenberg near Berlin and the netwoks was oriented by the astronomic azimuth of the triangle side connecting Rauenberg and Berlin-Marienkirche.

The German Reference Network (DREF91) has been carried out in April 1991 by GPS campaign, which comprises 109 sites. The standard deviation of the final set of threedimensional coordinates is 1 to $2 \mathrm{~cm}$ horizontal, $2 \mathrm{~cm}$ vertical. Figure 1 shows the distribution of DREF91.

The availability of the German Reference Network (DREF91) GPS campaign results makes it possible to analyze the transformation relationship between the German national coordinate system (DHDN, DHHN) and European Terrestrial Reference Frame 1989 (ETRF89).

\section{The 7 Parameter Similarity Transformation}

The 7 parameter similarity transformation between any two Cartesian systems, from $\left(X_{1}, Y_{1}, Z_{1}\right)$ to $(X, Y, X)$ can be written as

$$
\left(\begin{array}{c}
X \\
Y \\
Z
\end{array}\right)=\left(\begin{array}{c}
T_{1} \\
T_{2} \\
T_{3}
\end{array}\right)+\left(\begin{array}{ccc}
k & R_{z} & -R_{y} \\
-R_{z} & k & R_{x} \\
R_{y} & -R_{x} & k
\end{array}\right)\left(\begin{array}{c}
X_{1} \\
Y_{1} \\
Z_{1}
\end{array}\right)
$$

where $T_{1}, T_{2}, T_{3}$ are translate parameters; $R_{x}, R_{y}, R_{z}$ are differential rotation parameters; $k$ is scale parameter.

*Now at Geodetic Institute of Stuttgart University, 70174 Germany. On leave from Xiían Research Institute of Surveying and Mapping, China.

Copy right $(\mathrm{C})$ The Society of Geomagnetism and Earth, Planetary and Space Sciences (SGEPSS); The Seismological Society of Japan; The Volcanological Society of Japan; The Geodetic Society of Japan; The Japanese Society for Planetary Sciences.
The computer programs developed for this study are the main program CTP and two sub-program ENXYZ and GEOFRG for data preparation. An $L_{1}$-Norm method (Späth, 1987) is also developed here to check the gross error of the quasi-coordinates observations and compare the parameters.

\section{Data Preparation and the Solution of T-Para- meters}

3.1 National coordinate system (DHDN, DHHN) to ellipsoidal coordinates

The Gauss-Krueger coordinates of DHDN can be transformed to Bessel ellipsoidal coordinates Latitude (B) and longitude (L) through inverse conformal mapping formulas given by the series expansions in "Koordinaten auf geodaetischen Bezugsflaechen" by S. Heitz. The Bessel ellipsoidal height $h$ can be transformed from $H$ over Normal-Null surface in DHHN by

$$
h=H_{S}+N_{N N}
$$

where $H_{s}$ is the normal-orthometric (levelled) height and $N_{N N}$ is the distance from the Bessel Ellipsoid to the NormalNull surface. In practical calculation, the differences among $N N$ surface, quasigeoid and geoid, which are a few mm (flat terrain) and up to some $d m$ (mountainous area), are neglected (Torge, 1991; Niemeier, 1992; Ihde, 1995). So the following approximate equation is used instead of Eq. (2)

$$
h=H_{s}+\zeta
$$

where $\zeta$ is the height anomaly calculated by the gravimetric quasigeoid. For DHHN in the West German states the Denker88 model can be used (Denker, 1989). The newly published European Gravimetric Geoid model EGG97 has also been used in this study (Denker, 1998).

Both models are the quasigeoids, which refer to a geocentric system GRS80 and can be transformed subsequently 


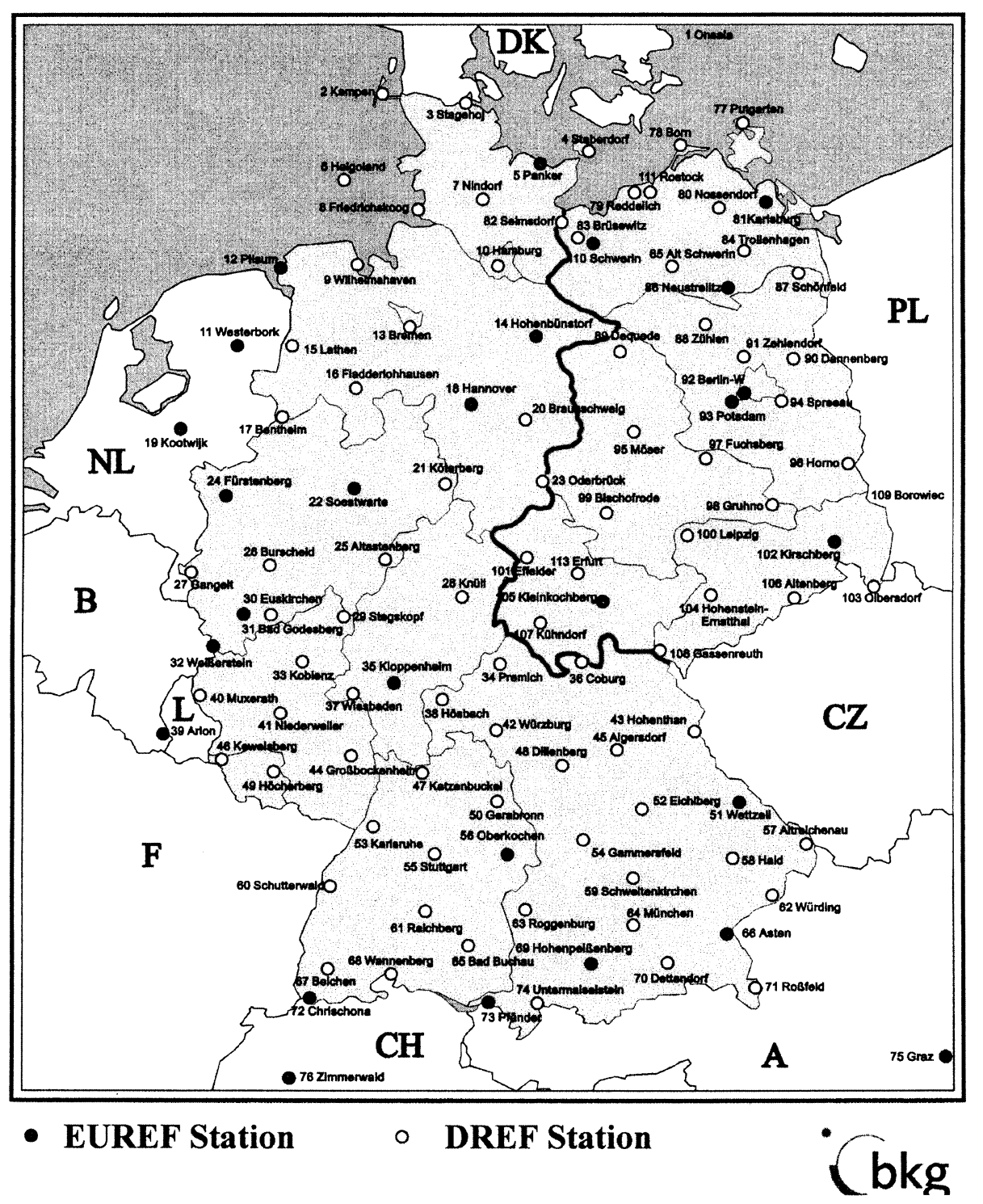

Fig. 1. German GPS-Reference Net (DREF 1991).

to other reference systems using input datum parameters in GEOFRG subroutine. In this study besides the original datum parameters derived through the Doppler-campaign (Rinner et al., 1982), another set of parameters published by IfAG is also used, which results in two sets of height anomaly related to the Bessel Ellipsoid surface. The difference of Denker and EGG97 height anomaly are provided in Fig. 7, from which it can be seen that the differences are systematically positive with mean value of $0.37 \mathrm{~m}$ and RMS of $\pm 0.15 \mathrm{~m}$. The abnormal difference of $-0.54 \mathrm{~m}$ occurs on the site 0710 , where the geodetic height is up to $1530 \mathrm{~m}$. This may be attributed to some improper determination of Denker88 model in this area.

The transformation parameter solution using above two set reductions are listed in Tables 1.1 and 1.2, which indicate that the both geoidal models result in almost the same global parameters. The obvious difference is $1.924 \mathrm{~m}$ between translation parameters of axis $Y$ and corresponding transformation residuals are in the same level. The difference between the two models and comparison with GPS geoidal height will be discussed in Subsection 4.3.

3.2 Bessel ellipsoidal coordinates $B, L, h$ to geodetic Cartesian coordinates $X, Y, Z$

Conversion of ellipsoidal coordinates to cartesian coordinates and the reverse conversion are accomplished through the general formula as found, for example, in Rapp (1984).

3.3 The construction of quasi-observations and solutions

The original data are "Endgueltige Koordinaten EUREF und DREF in ETRF 89 Epoche 89.0", which include DREF91 Geocentric Coordinate, DREF91 Geographic Coordinate and DREF91 Gauss-Krueger-Coordination (BesselEllipsoid). For lack of quasigeoid data to System 42/83, HN 76, we have only used 69 colocated sites in the area of West Germany to estimate the 7 transformation parameters between DHDN and ETRF89, in which the accuracy of these two set Cartesian coordinates are $\pm 0.1 \mathrm{~m}$ for DHDN and $\pm 1 \sim 2 \mathrm{~cm}$ for DREF91 and are used to calculate the weights. The quasi-observations are prepared with methods mentioned above and with different quasigeoid anomaly 
Table 1. Comparison of transformation parameters from DHDN to ETRF.

1.1 DHDN $\rightarrow$ ETRF (using Quasigeoid anomalies from EGG97 model and transformed with IfAG and Doppler parameters in GEOFRG program

\begin{tabular}{|c|c|c|c|c|c|c|c|c|c|}
\hline Solution & $T_{1}(\mathrm{~m})$ & $T_{2}(\mathrm{~m})$ & $T_{3}(\mathrm{~m})$ & $R_{x}\left({ }^{\prime \prime}\right)$ & $R_{y}\left({ }^{\prime \prime}\right)$ & $R_{z}\left({ }^{\prime \prime}\right)$ & $d m\left(\times 10^{-6}\right)$ & $V_{\max }(\mathrm{cm})$ & $\sigma_{0}(\mathrm{~cm})$ \\
\hline WD69-1 & 582.6235 & 109.1885 & 412.5300 & -1.1416 & -0.3126 & 3.1881 & 8.3586 & & 6.54 \\
\hline (IfAG) & \pm 2.2951 & \pm 3.6669 & \pm 2.0221 & \pm 0.0986 & \pm 0.0781 & \pm 0.0903 & \pm 0.2907 & \pm 247.41 & $\mathrm{WRMS}=64.37$ \\
\hline WD69-2 & 592.7775 & 74.1605 & 409.0986 & -0.2730 & -0.0120 & 2.4461 & 8.2591 & & 6.76 \\
\hline (Doppler) & \pm 2.3703 & \pm 3.7870 & \pm 2.0883 & \pm 0.1018 & \pm 0.0806 & \pm 0.0933 & \pm 0.3002 & \pm 266.46 & $\mathrm{WRMS}=66.48$ \\
\hline \multicolumn{10}{|c|}{ 1.2 DHDN $\rightarrow$ ETRF (using quasigeoid anomalies from Denker88 model and transformed with IfAG and Doppler parameters in GEOFRG program } \\
\hline WD69-3 & 581.3386 & 107.2638 & 413.8739 & -1.0887 & -0.3719 & 3.1537 & 8.4134 & & 6.58 \\
\hline IfAG & \pm 2.3066 & \pm 3.6852 & \pm 2.0322 & \pm 0.0991 & \pm 0.0785 & \pm 0.0908 & \pm 0.2921 & \pm 246.34 & $\mathrm{WRMS}=64.69$ \\
\hline WD69-4 & 591.4778 & 72.3179 & 410.4429 & -0.2221 & -0.0478 & 2.4133 & 8.3137 & & 6.79 \\
\hline Doppler & \pm 2.3798 & \pm 3.8022 & \pm 2.0967 & \pm 0.1022 & \pm 0.0810 & \pm 0.0936 & \pm 0.3014 & \pm 254.14 & $\mathrm{WRMS}=66.75$ \\
\hline \multicolumn{10}{|c|}{$1.3 \quad$ DHDN $\rightarrow$ ETRF $\left(L_{2}\right.$-Norm and $L_{1}$-Norm Solutions $)$} \\
\hline WD69-1 $\left(L_{2}\right)$ & 582.6235 & 109.1885 & 412.5300 & -1.1416 & -0.3126 & 3.1881 & 8.3586 & 247.41 & 6.54 \\
\hline WD69-5 $\left(L_{1}\right)$ & 584.5322 & 107.7706 & 413.5374 & -1.2693 & -0.3137 & 2.9606 & 8.0704 & 327.82 & $L_{1}-\mathrm{Norm}=99.04 \mathrm{~m}$ \\
\hline \multicolumn{10}{|c|}{ 1.4 The published transformation parameters which are used in the GEOFRG program } \\
\hline IfAG & 582.0000 & 105.0000 & 414.0000 & -1.0400 & -0.3500 & 3.0800 & 8.3000 & \multicolumn{2}{|c|}{$(\mathrm{ZfV} 4 / 95)$} \\
\hline Doppler & 583.0000 & 68.2000 & 399.1000 & 0.0000 & 0.0000 & 2.5460 & 10.5200 & \multicolumn{2}{|c|}{ (DGK R. B260/82) } \\
\hline
\end{tabular}

models.

The transformation parameters are solved with $L_{2}$ - and $L_{1}$ Norm methods for the entire area of West Germany, every single state and some regional areas. Detailed solutions are listed in Table 1.3.

\section{The Analysis of the Transformation Parameters}

4.1 The analysis of global transformation parameters and corresponding residuals

The different solutions are listed in Table 1. The first solution WD69-1 using 69 colocated sites for the area of all West Germany indicates that there are a relatively larger translations between DHDN reference frame and ETRF, which are $582.6235,109.1885$ and $412.5300 \mathrm{~m}$ in $X, Y$ and $Z$ dimension. The estimated Euler rotations are in the order of arc second with the largest value of $3.2^{\prime \prime}$ around $Z$ axis. This shows that the DHDN has an orientation uncertainty. The scale difference of $8.4 \mathrm{ppm}$ also verifies the fact that the DHDN has a scale variation up to $10 \mathrm{ppm}$ (Torge, 1991).

The accuracy of estimated transformation parameters can be represented with standard deviations $\Sigma_{x i i}$ from the covariance matrix

$$
\Sigma_{X}=\sigma_{0}^{2} \cdot\left(\mathbf{A}^{T} \mathbf{P} A^{-1}\right)
$$

and the weighted root mean square (WRMS) residuals

$$
\mathrm{WRMS}=\sqrt{\left(\sum_{i} \frac{V_{X i}^{2}}{\sigma_{X i}^{2}}\right) /\left(\sum_{i} \frac{1}{\sigma_{X i}^{2}}\right)}
$$

The standard deviations listed below these parameters of WD69-1 exhibits that the uncertainties of estimated translation parameters are $2.0 \sim 3.65$ meters, which will bring the corresponding transformation accuracy of Cartesian coordinates with the same order and result in about \pm 5 meters position error. The WRMS residual of colocated coordinates is 0.64 meters with the largest residual of 2.47 meters, which occurs on the dimension $y$ of site $0030 . L_{1}$-Norm solution points out that the same dimension $y$ of site 0030 has the largest residual of 3.28 meters.

From the horizontal distribution of residuals shown in Fig. 2 we can see that horizontal position residuals of these sites bordering the boundary of DHDN, except these sites in right middle area, are in the range of 1.5 to 2.0 meters and larger than these inner sites. The two largest residuals occur in site 0020 and 0030 in the northern boundary, which are 2.95 and 2.49 meters respectively.

From the direction of the horizontal residual vectors we can obviously find that there are two rotational trends, which are counter-clockwise in the northern part and clockwise in the southern part. The similar two opposite direction rotations of residuals also occur in the independent solution in the northern and southern part (see Fig. 3). Some published maps of transformation residuals have similar rotations (Vermeer, 1995). These examples show that two opposite direction rotations do not come from the colocated coordinates, but from three-dimension similarity transformation (1) itself.

The vertical residuals shown in Fig. 4 are relative smaller than the plane position residuals, which range from $-0.15 \mathrm{~m}$ to $+0.18 \mathrm{~m}$. An abnormal value of $-0.32 \mathrm{~m}$ occurs on site 0060. The reason will be discussed further in Subsection 4.3.

In summary the positional residuals are provided in Fig. 5, which has almost the same magnitude and distribution as the dominant plane positional residuals. The accuracy of estimated transformation parameters and the magnitude of transformation residuals essentially depend on the accuracy of colocated coordinates. While the accuracy of DREF91 coordinate in the level of $1 \sim 2$ centimeters, it is shown that the DHDN is the main error source to the accuracy of estimation and corresponding residuals. The similarity transformation solutions verify the fact that the DHDN was established 


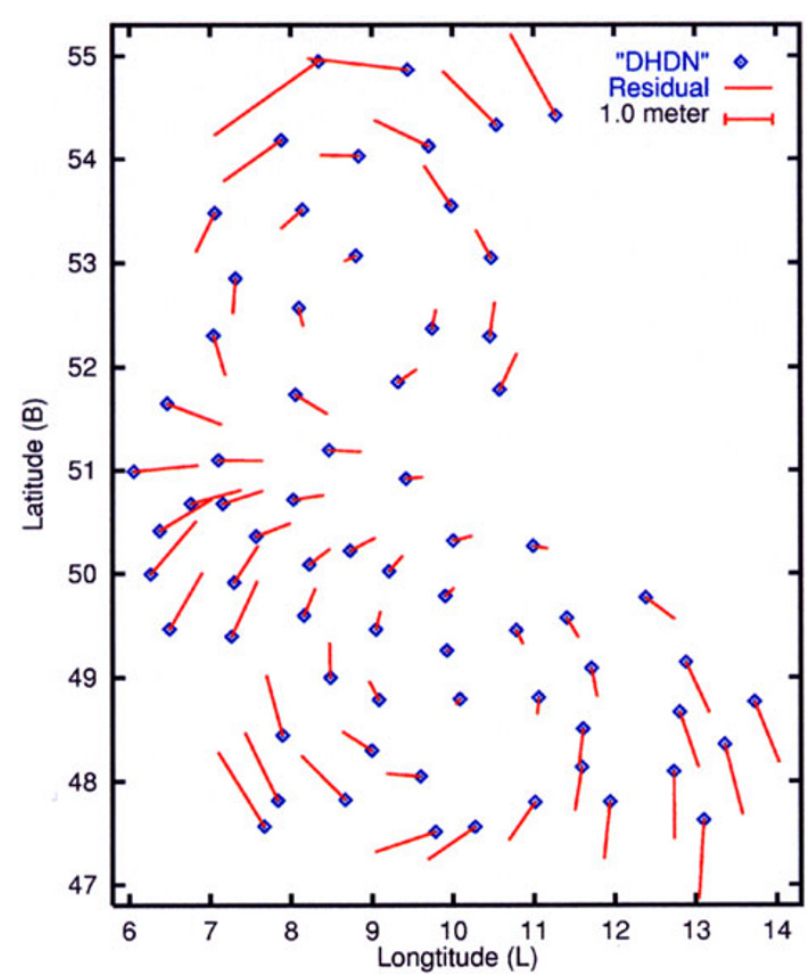

Fig. 2. Residuals in B and L for the area of West Germany.

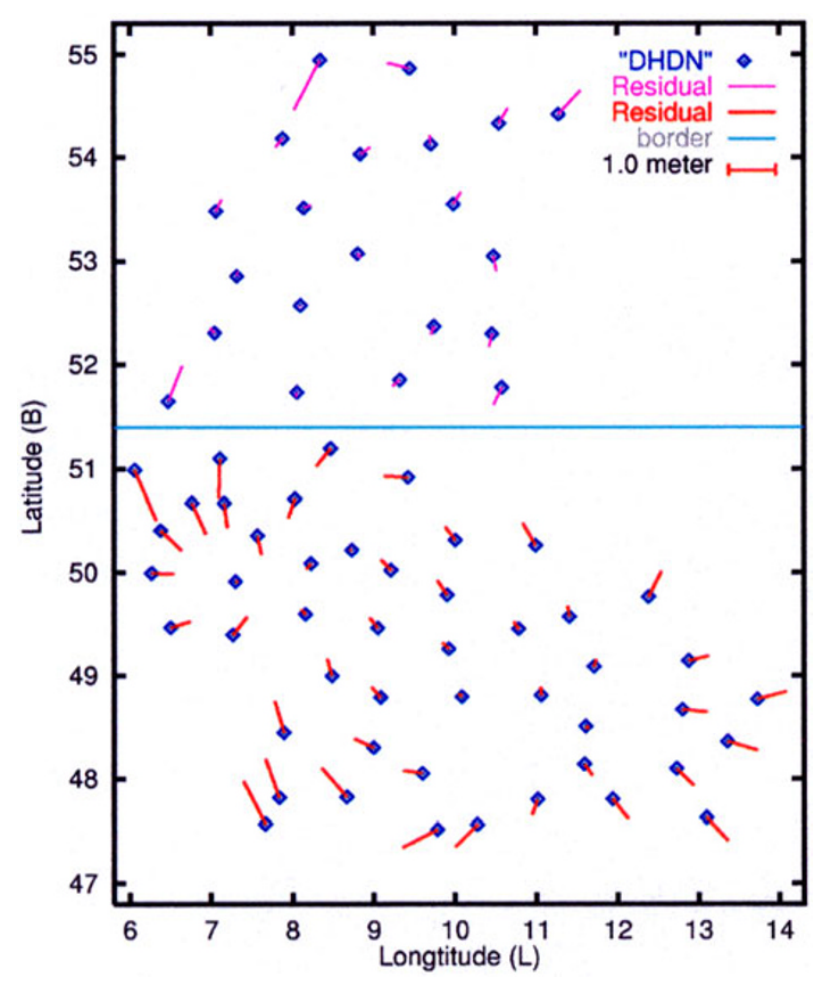

Fig. 3. Residuals in B and $\mathrm{L}$ for the northern and southern parts.

with different triangulation chains and nets and processed by "development method" (Torge, 1991) in the 80-year period (1870 to 1950), which made the DHDN inhomogeneous and resulted in global horizontal position accuracy of 1.5 to $2 \mathrm{~m}$,

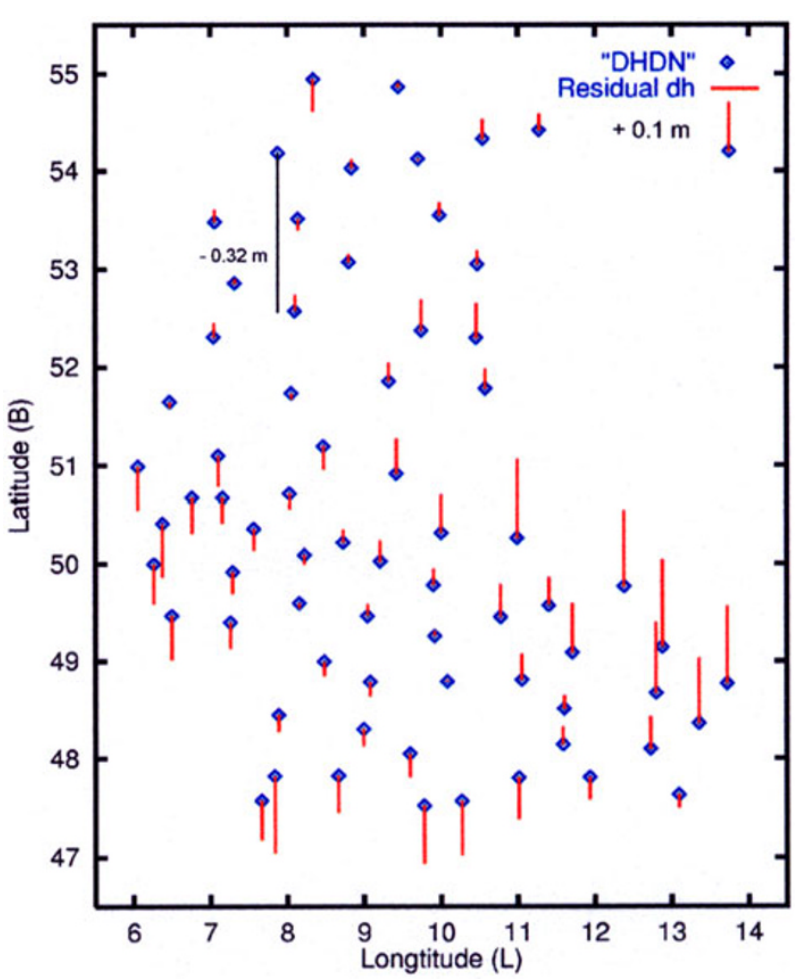

Fig. 4. Height residuals for the area of West Germany.

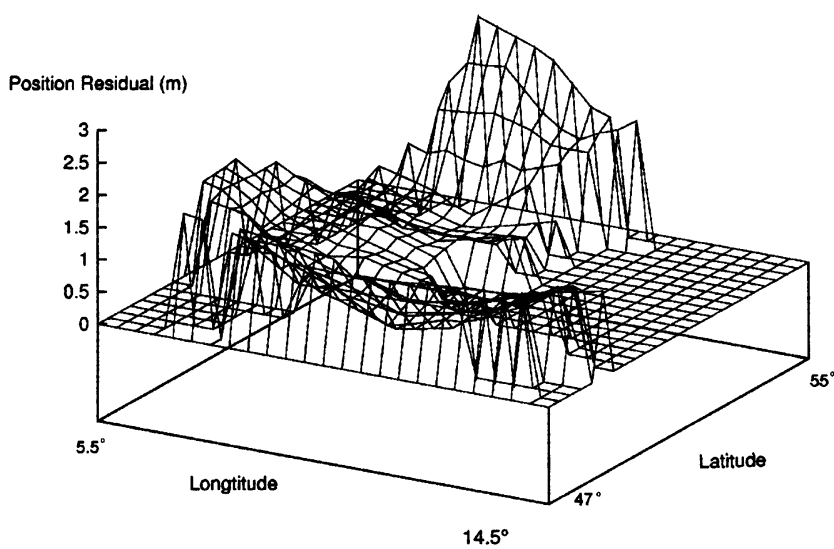

Fig. 5. The Positional Residuals of Transformation from DHDN to ETRF89.

regional accuracy of $0.1 \mathrm{~m}$.

For further verification, the solution of parameters $L_{1}$ Norm methods are also used for the same data. From the estimated results listed on Table 1.3 we can see that there are small differences between $L_{1}$ and $L_{2}$-Norm solutions. For the inhomogeneity of DHDN it is difficult to verify that these sites with larger residuals have gross errors. The detection of gross errors can be performed in the independent solution of each single state.

4.2 The analysis of regional parameters and corresponding residuals

Using the same colocated data sets, the transformation parameters for each single state of West Germany are estimated independently. The sites 0460 and 0490 in the State of Saar- 
Table 2. Transformation parameters for every single state and region.

\begin{tabular}{|c|c|c|c|c|c|c|c|c|c|}
\hline Solution & $T_{1}(\mathrm{~m})$ & $T_{2}(\mathrm{~m})$ & $T_{3}(\mathrm{~m})$ & $R_{x}\left({ }^{\prime \prime}\right)$ & $R_{y}\left({ }^{\prime \prime}\right)$ & $R_{z}\left({ }^{\prime \prime}\right)$ & $d m\left(\times 10^{-6}\right)$ & $V_{\max }(\mathrm{cm})$ & WRMS $(\mathrm{cm})$ \\
\hline SH8 & \pm 11.8526 & \pm 8.9841 & \pm 9.9084 & \pm 0.2760 & \pm 0.4365 & \pm 0.2624 & \pm 1.1751 & 64.74 & \pm 23.31 \\
\hline NS10 & 0.9475 & 0.6279 & 0.7697 & 0.0186 & 0.0358 & 0.0188 & 0.0805 & 5.80 & 2.46 \\
\hline NRW9 & 5.5921 & 5.9118 & 5.2749 & 0.1724 & 0.2217 & 0.1411 & 0.5416 & 31.55 & 12.53 \\
\hline HE3 & 18.5359 & 38.6239 & 20.1969 & 1.0312 & 0.8495 & 0.7555 & 1.0432 & 10.39 & 4.54 \\
\hline RLP7 & 3.2583 & 4.1847 & 3.2026 & 0.1198 & 0.1205 & 0.1069 & 0.4132 & 12.79 & 6.15 \\
\hline BW11 & 2.5505 & 3.9187 & 2.6720 & 0.1088 & 0.0984 & 0.0945 & 0.3237 & 20.69 & 8.45 \\
\hline BA21 & 1.8332 & 2.0422 & 1.6452 & 0.0575 & 0.0656 & 0.0561 & 0.2166 & 53.19 & 12.72 \\
\hline \multicolumn{10}{|c|}{ 2.2 Comparision of the similarity among these solutions by the scale difference } \\
\hline Solution & $\mathrm{BA}$ & $\mathrm{SH}$ & NS & BW & $\mathrm{HE}$ & NRW & RLP & & \\
\hline$d m\left(\times 10^{-6}\right)$ & 5.4197 & 7.0547 & 9.1190 & 9.5488 & 10.4266 & 13.6720 & 13.3859 & & \\
\hline \multicolumn{10}{|c|}{ 2.3 Comparision of the similarity among these solutions by the Euler rotation $R_{z}$} \\
\hline Solution & BW & BA & RLP & $\mathrm{HE}$ & NRW & NS & SH & & \\
\hline Aver. Latitude & $48.43^{\circ}$ & $49.08^{\circ}$ & $49.90^{\circ}$ & $50.70^{\circ}$ & $51.43^{\circ}$ & $51.85^{\circ}$ & $54.12^{\circ}$ & & \\
\hline$R_{z}\left({ }^{\prime \prime}\right)$ & 2.0055 & 2.5058 & 2.5391 & 2.9509 & 3.1178 & 4.1241 & 4.5911 & & \\
\hline
\end{tabular}

land are included in the solution of the State of RheinlandPfalz (RLP), the site 0130 in the State of Bremen and site 0100 in Hamburg are included in solution of the State of Niedersachen (NS) and Schleswig-Holstein (SH) respectively. The estimated parameters of these states are different from each other. The corresponding horizontal position residuals of colocated sites shown in Fig. 6 exhibit (1) that the residuals for each single state are far smaller than these of the global solution. The largest residual of $0.75 \mathrm{~m}$ occurs on the site 0020 ; (2) these points bordering the boundary of every state still have the larger residuals than the inner sites. These residuals and their WRMS value, which are from 2.46 $\mathrm{cm}$ to $23.31 \mathrm{~cm}$ verify that the regional solutions have better fitting than the global solution.

In Table 2.2 the solutions are listed according to the value of scale difference parameters $d m$, which vary from $5.4 \mathrm{ppm}$ to $13.67 \mathrm{ppm}$. This shows that there are systematic differences in defined and observational scales in every state. The agreement between the scale parameters in NRW and RLP demonstrates that the Netz 77 and Netz 80 were established with the same datum.

From the value of translation and scale parameters an approximate relationship among each state can be found:

$$
\begin{aligned}
\left(N_{i}\right. & \left.\cdot d m_{i}+\left(\sqrt{T_{1}^{2}+T_{2}^{2}+T_{3}^{2}}\right)_{i}\right) \\
& \approx\left(N_{j} \cdot d m_{j}+\left(\sqrt{T_{1}^{2}+T_{2}^{2}+T_{3}^{2}}\right)_{j}\right) \\
& \approx 775 \mathrm{~m}
\end{aligned}
$$

where $N_{i}$ is the radius of curvature in the prime vertical. If the value of scale parameter increases the value of translations will decrease.

The next trends can be seen from Table 2.3: the Euler rotation $R_{z}$ of each state are associated with the average latitude

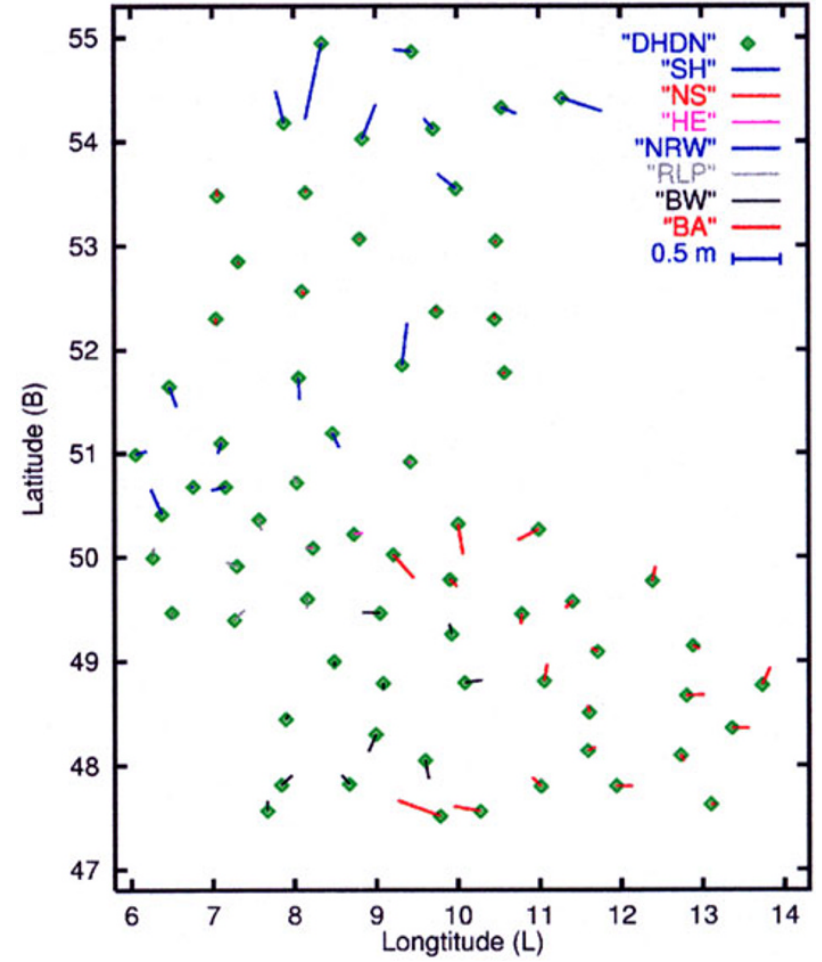

Fig. 6. Residuals in B and L for the each single state (independent solution).

of the sites in it, which reflects the effect from local Laplace azimuth correction in the form

$$
\begin{aligned}
R_{Z i} & =\sin B_{i} \cdot d A_{i} \\
d A_{i} & =\frac{\cos B_{0}}{\cos B_{1}} \cdot \cos \left(L_{i}-L_{0}\right) \cdot d A_{0}
\end{aligned}
$$

where the $B_{0}, L_{0}$, and $d A_{0}$ are the coordinates and Laplace 
azimuth correction of DHDN at the origin point in Rauenberg. Note the published value of $d A_{0}$ is $2.96^{\prime \prime}$.

Among these states the best solution occurs in the State of Niedersachsen, in which the standard deviations of translation parameters are smaller than one meter and the transformation residuals are the smallest with the largest value of $6 \mathrm{~cm}$ and WRMS residual of 2.46 centimeters. This best fitting should be attributed to the homogeneous controlpoint-field which is derived from a complete new adjustment (Lagestatus_100) to the new observations of DHDN by microwave EDM methods in this state (Fröhlich, 1998).

It should be pointed out that the standard deviation of transformation parameters is not only decided by the quality of colocated coordinates, but also by the number of colocated sites. For example the standard deviation of estimated parameters for the State of Hessen are in the range of 18 to 38 meters. This does not mean that the accuracy of transformation in this area is also in the same range. Theoretically, the higher the number of colocated sites, the more accurate the transformation parameters between two coordinate systems can be reached. Since the DHDN is inhomogeneous, we have to solve the transformation parameters in regional area to get better fitting and enough accuracy. Such a transformation relationship is not between DHDN and ETRF, it is only between regional system and ETRF. The so-called global transformation parameters between DHDN and ETRF are only in average sense and can consequently be used for relatively lower accuracy calculation and applications.

\section{Conclusion and Suggestion}

Analyses and comparisons for global and regional transformation described above confirm that

- The accuracy of the global transformation between DHDN and ETRF is $\pm 5 \mathrm{~m}$ with WRMS of $\pm 0.64 \mathrm{~m}$. Because of the fact of inhomogeneity of DHDN, the global transformation parameters are only indicative in average sense and can consequently be used for the succeeding transformation of lower accuracy and related applications.

- Regional solutions show much better fitting, in which the WRMS values vary from $2.46 \mathrm{~cm}$ to $23.31 \mathrm{~cm}$. These transformation relationships are not between DHDN and ETRF, they are only between regional sys- tems and ETRF. The comparison of scale differences and parameters Euler rotation parameters $R_{z}$ of each state reveal the systematic differences in definition and observation among regional systems of DHDN.

According to the results of this study it is suggested that

- To process a new adjustment of DHDN with the control of DREF91 points.

- Since many existing geodetic and mapping products continue to be based on DHDN coordinate system, datum transformations will continue to be useful.

Acknowledgments. The author thanks Dr. E. C. Pavlis and an anonymous reviewer for their constructive comments, which helped to clarity some of points in this paper. Prof. Dr. W. Benning supported this research and proofread the drafting paper. Dr. J. Ihde has provided the basic distribution data and detail precision of DHDN and DREF91 and useful suggestion. Dr. Heiner Denker, has kindly supplied the EGG97 height anomalies to the author. All this support is gratefully acknowledged.

\section{References}

Denker, H., A new Gravimetric Quasigeoid for the Ferderal Republic of Germany, Deutsche Geodätische Kommission, Reihe B, Nr. 291, München, 1989.

Denker, H., The quasigeoid anomaly data from EGG97, personal communication, 1998.

Fröhlich, H., et al., Praxisnahe 3D-kartesische 7-Parameter-Transformation für Anwendungen in der Landesvermessung-Das Programm TRANSSIB — , Vermessungswesen und Raumordnung, S 146-151, 1998.

Heitz, S., Koordinaten auf geodaetischen Bezugsflaechen, Dümmler Verlag Bonn, 1985.

Hofmann-Wellenhof, B., et al., Global Positioning System-Theory and Practice, third revised edition, Springer-Verlag Wien-New York, 1994.

Ihde, J. and W. Lindstrot, Datumstransformation zwischen den Bezugssystemen ETRF/WGS, DHDN und System 42, ZfV, Nr. 4, S.192-196, 1995.

Niemeier, W., Zur Nutzung von GPS-Meflergebnissen in Netzen der Landesund Ingenieurvermessung, ZfV, Nr. 8/9, S.542-556, 1992.

Rapp, R. H., Geometric Geodesy, Part I, Department of Geodetic Science, The Ohio State University, Columbus, Ohio, September, 1984.

Rinner, K., et al., Die Deutsch-Österreichische Dopplerkampagne, DGK, B Nr. 260, München, 1982.

Späth, H., Mathematische Software zur linearen Regression, R. Oldenbourg Verlag Munchen Wien, 1987.

Torge, W., Geodesy, W. de Gruyter, Berlin-New York, 1991.

Vermeer, M. (editor), Coordinate System, GPS and the Geoid, Reports of the Finnish geodetic Institute, 1995.

J. Cai (e-mail: cai@gia.rwth-aachen.de) 